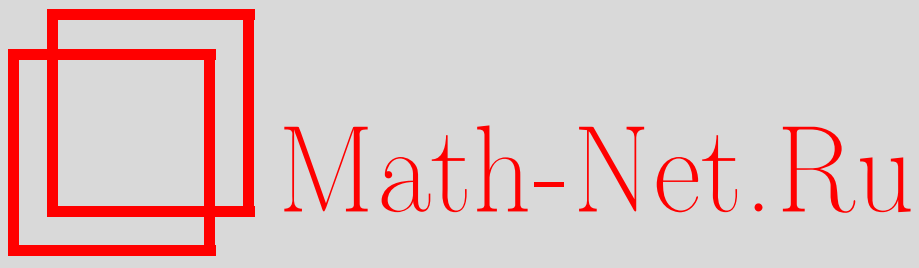

A. I. Budkin, On the quasivarieties generated by a finite group and lacking any independent bases of quasi-identities, Sibirsk. Mat. Zh., 2020, Volume 61, Number 6, 1234-1246

DOI: https://doi.org/10.33048/smzh.2020.61.603

Use of the all-Russian mathematical portal Math-Net.Ru implies that you have read and agreed to these terms of use http://www . mathnet.ru/eng/agreement

Download details:

IP: 34.227 .88 .159

April 26, 2023, 13:01:23

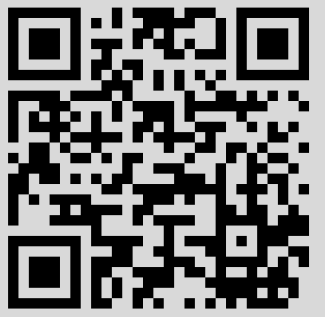


Сибирский математический журнал

Ноябрь-декабрь, 2020. Том 61, № 6

УДК 512.54

\title{
О КВАЗИМНОГООБРАЗИЯХ, ПОРОЖДЕННЫХ КОНЕЧНОЙ ГРУППОЙ И НЕ ИМЕЮЩИХ НЕЗАВИСИМЫХ БАЗИСОВ КВАЗИТОЖДЕСТВ
}

\section{А. И. Будкин}

\begin{abstract}
Аннотация. Пусть $\mathscr{R}_{p^{k}}-$ многообразие 2-ступенно нильпотентных групп экспоненты $p^{k}$ с коммутантом экспоненты $p$ ( $p-$ простое число). Доказано, что множество подквазимногообразий $\mathscr{R}_{p^{k}}(k \geq 2)$, порожденных конечной группой и не имеющих независимых базисов квазитождеств, бесконечно.
\end{abstract}

DOI 10.33048/smzh.2020.61.603

Ключевые слова: квазимногообразие, квазитождество, независимый базис, нильпотентная группа.

\section{Введение}

В работе рассматривается вопрос о существовании независимых базисов квазитождеств групп и исследуется сложность решеток квазимногообразий групп. Изучению независимых базисов квазитождеств групп посвящено значительное количество работ. Приведем некоторые из них. В [1] доказано, что если квазимногообразие групп содержит бесконечную циклическую группу и не содержит бесконечного множества групп простого порядка, то оно имеет независимый базис квазитождеств. Ввиду этого для квазимногообразий групп без кручения рассматривается задача о существовании независимого базиса квазитождеств в классе групп без кручения. В [2] найдены условия существования независимого базиса квазитождеств в классе групп без кручения. В частности, оказалось, что некоторые широко изучаемые квазимногообразия (например, квазимногообразие, порожденное неабелевой свободной разрешимой группой, всех линейно упорядочиваемых групп) имеют независимые базисы квазитождеств в классе групп без кручения. В [3] показано, что множество квазимногообразий разрешимых групп, не имеющих независимого базиса квазитождеств в классе групп без кручения, имеет мощность континуума. В [4] построены континуальные серии квазимногообразий нильпотентных групп, не имеющих независимых базисов квазитождеств. В [1] построено квазимногообразие групп, не имеющее независимого базиса квазитождеств, которое можно задать независимой системой $\forall$-формул.

В [5] доказано, что свободная 2-нильпотентная группа ранга $n \geq 2$ не имеет независимого базиса квазитождеств в классе групп без кручения. В [6] также показано, что аналогичным свойством обладает квазимногообразие, порожденное неабелевой группой порядка $p^{3}$, где $p-$ простое число, $p \neq 2$. Пусть $\mathscr{R}_{p^{k}}$ многообразие 2-ступенно нильпотентных групп экспоненты $p^{k}$ с коммутантом экспоненты $p$ ( $p$ - простое число, $k \geq 2$ ). Из [7] следует, что квазимногообразие,

(c) 2020 Будкин А. И. 
порожденное свободной неабелевой $\mathscr{R}_{p^{k}}$-группой, не имеет независимого базиса квазитождеств.

В процессе развития теории квазимногообразий достаточно быстро стало известно, что решетки квазимногообразий имеют весьма сложное строение. На сложность решеток квазимногообразий универсальных алгебр указывают, например, статьи [8-11]. Информацию о сложности решеток квазимногообразий групп можно найти в [7,12-15]. Отметим работы $[13,14,16]$, в которых установлено, что лишь одно неабелево квазимногообразие 2-ступенно нильпотентных групп без кручения и только конечное множество квазимногообразий 2ступенно нильпотентных групп экспоненты $p^{k}$ с коммутантом простой экспоненты имеют конечную решетку подквазимногообразий.

Нами получена теорема, характеризующая сложность решетки подквазимногообразий квазимногообразия $\mathscr{R}_{p^{k}}(k>1)$, а именно установлено, что существует бесконечное множество подквазимногообразий $\mathscr{M} \subseteq \mathscr{R}_{p^{k}}$, порожденных конечной группой, таких, что для любого квазимногообразия $\mathscr{N}(\mathscr{M} \subsetneq \mathscr{N} \subseteq$ $\left.\mathscr{R}_{p^{k}}\right)$ промежуток $[\mathscr{M}, \mathscr{N}]$ в решетке квазимногообразий континуален. С учетом этого результата установлено, что множество квазимногообразий, порожденных конечной группой, содержащихся в $\mathscr{R}_{p^{k}}(k \geq 2)$ и не имеющих независимых базисов квазитождеств, бесконечно.

\section{$\S 1$. Предварительные замечания}

Отметим, что необходимую информацию о квазимногообразиях можно найти в $[16,17]$. Введем следующие определения и обозначения.

$\langle S\rangle$ - группа, порожденная множеством $S,\langle a\rangle$ - циклическая группа, порожденная элементом $a, G^{\prime}$ - коммутант группы $G, Z(G)$ - центр $G, \operatorname{ker} \varphi-$ ядро гомоморфизма $\varphi$. Если $x, y$ - элементы группы, то $[x, y]=x^{-1} y^{-1} x y$. Как обычно, если $A, B$ - подгруппы группы $G$, то $[A, B]=\langle[a, b] \mid a \in A, b \in B\rangle$.

$\mathscr{R}_{p^{k}}(p-$ простое число, $k \geq 2)-$ многообразие, заданное тождествами

$$
(\forall x)(\forall y)(\forall z)([x, y, z]=1), \quad(\forall x)\left(x^{p^{k}}=1\right), \quad(\forall x)(\forall y)\left([x, y]^{p}=1\right),
$$

$q G$ - квазимногообразие, порожденное группой $G$,

$\mathscr{M}(G)$ - наименьшая нормальная подгруппа группы $G$, фактор-группа по которой принадлежит квазимногообразию $\mathscr{M}$,

$[\mathscr{M}, \mathscr{N}]=\{\mathscr{R} \mid \mathscr{M} \subseteq \mathscr{R} \subseteq \mathscr{N}\}-$ замкнутый промежуток в решетке квазимногообразий групп,

$\checkmark$ - решеточная сумма,

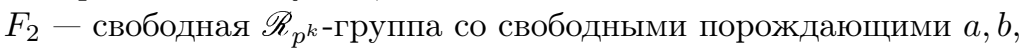

$Z_{p^{n}}$ - циклическая группа порядка $p^{n}$.

Будем пользоваться тем, что в любой 2-ступенно нильпотентной группе истинны тождества

$$
(\forall x)(\forall y)(\forall z)([x y, z]=[x, z][y, z]), \quad(\forall x)(\forall y)(\forall z)([x, y z]=[x, y][x, z]) .
$$

Будем говорить, что коммутирование на нецентральных элементах группы $G$ транзитивно, если для любых $x, y, z \in G$, не содержащихся в центре $G$, из $[x, y]=1,[x, z]=1$ следует $[y, z]=1$.

Предположим, что группы $A$ и $B$ имеют в $\mathscr{R}_{p^{k}}$ следующие представления:

$$
A=\left\langle\left\{x_{i} \mid i \in I_{1}\right\} ;\left\{t_{j}=1 \mid j \in J_{1}\right\}\right\rangle, \quad B=\left\langle\left\{y_{i} \mid i \in I_{2}\right\} ;\left\{r_{j}=1 \mid j \in J_{2}\right\}\right\rangle,
$$


в непересекающихся множествах порождающих символов. Тогда группа, имеющая в $\mathscr{R}_{p^{k}}$ представление

$$
\left\langle\left\{x_{i} \mid i \in I_{1}\right\} \cup\left\{y_{i} \mid i \in I_{2}\right\} ;\left\{t_{j}=1 \mid j \in J_{1}\right\} \cup\left\{r_{j}=1 \mid j \in J_{2}\right\}\right\rangle,
$$

называется свободным произведением в $\mathscr{R}_{p^{k}}$ групп $A$ и $B$ и обозначается через $A * \mathscr{R}_{p^{k}} B$. Часто вместо $A * \mathscr{R}_{p^{k}} B$ будем писать $A * B$, опуская индекс $\mathscr{R}_{p^{k}}$. Отметим, что множество порождающих символов группы $A *_{\mathscr{R}^{k}} B$ совпадает с объединением множеств порождающих символов групп $A$ и $B$, а множество ее определяющих соотношений - с объединением множеств определяющих соотношений этих групп.

Будем пользоваться следующей теоремой Дика [18, п. 11.2, теорема 5].

Лемма 1. Пусть группа $A$ имеет в данном квазимногообразии $\mathscr{M}$ представление

$$
A=\left\langle\left\{x_{i} \mid i \in I\right\} ;\left\{r_{j}\left(x_{j_{1}}, \ldots, x_{j_{l(j)}}\right)=1 \mid j \in J\right\}\right\rangle .
$$

Предположим, что $H \in \mathscr{M}$ и группа $H$ содержит множество элементов $\left\{g_{i} \mid i \in\right.$ $I\}$ такое, что для всякого $j \in J$ равенство $r_{j}\left(g_{j_{1}}, \ldots, g_{j_{l(j)}}\right)=1$ истинно в $H$. Тогда отображение $x_{i} \rightarrow g_{i}(i \in I)$ продолжается до гомоморфизма $A$ в $H$.

Ниже понадобится следующий признак принадлежности конечно определенной группы $G$ квазимногообразию $q \mathscr{R}$, порожденному классом $\mathscr{R}[16$, теорема 2.3.9; 17, следствие 2.1.21].

Лемма 2. Конечно определенная в квазимногообразии $\mathscr{N}$ группа $G$ принадлежит квазимногообразию, порожденному классом групп $\mathscr{R}(\mathscr{R} \subseteq \mathscr{N})$, тогда и только тогда, когда для любого элемента $g \in G(g \neq 1)$ существует гомоморфизм $\varphi_{g}$ группы $G$ в некоторую группу из класса $\mathscr{R}$ такой, что $g^{\varphi_{g}} \neq 1$.

Лемма 3. Пусть $G \in \mathscr{R}_{p^{k}}-$ конечная неединичная группа. Тогда группу $G$ в $\mathscr{R}_{p^{k}}$ можно задать представлением вида

$$
G=\left\langle x_{1}, \ldots, x_{n} ; x_{1}^{p^{n_{1}}} c_{1}=1, \ldots, x_{l}^{p^{n_{l}}} c_{l}=1, c_{l+1}=1, \ldots, c_{s}=1\right\rangle
$$

где $c_{1}, \ldots, c_{s}$ - элементы коммутанта свободной группы, $n_{i} \geq 1, i=1, \ldots, l$ (соотношения $x_{1}^{p^{n_{1}}} c_{1}=1, \ldots, x_{l}^{p^{n_{l}}} c_{l}=1$ либо $c_{l+1}=1, \ldots, c_{s}=1$ могут отсутствовать).

ДоказАтельство. Группа $G / G^{\prime}$ разлагается в прямое произведение своих неединичных циклических подгрупп: $G / G^{\prime}=\left\langle x_{1} G^{\prime}\right\rangle \times \cdots \times\left\langle x_{n} G^{\prime}\right\rangle$. Элементы $x_{1}, \ldots, x_{n}$, порождающие $G$ по модулю коммутанта, порождают $G$. В этих порождающих $G$ имеет требуемое представление. Лемма доказана.

Лемма 4. Пусть $\mathscr{M} \subseteq \mathscr{R}_{p^{k}}-$ произвольное квазимногообразие групп такое, что $Z_{p} * Z_{p} \in \mathscr{M}$. Предположим, что группы $A$ и $B$ из $\mathscr{M}$ в порождающих $a_{1}, \ldots, a_{n}$ и $b_{1}, \ldots, b_{m}$ соответственно имеют в $\mathscr{R}_{p^{k}}$ представления, аналогичные представлению из формулировки леммы 3. Если $N$ - подгруппа (возможно, единичная) группы $A * B$, порожденная некоторыми коммутаторами вида $\left[a_{i}, b_{j}\right]$, то $(A * B) / N \in \mathscr{M}$.

Доказательство. Пусть $G=(A * B) / N,[A, B]=\left\langle\left[a_{i}, b_{j}\right]\right| i=1, \ldots, n, j=$ $1, \ldots, m\rangle$. Так как $G /[A, B] \cong A \times B$, то $G /[A, B] \in \mathscr{M}$. Возьмем произвольный неединичный элемент $g \in[A, B]$. Достаточно (ввиду леммы 2) показать, что $g^{\varphi} \neq 1$ для некоторого гомоморфизма $\varphi$ группы $G$ в подходящую группу из $\mathscr{M}$. 
Элемент $g$ можно записать в виде

$$
g=\prod\left[a_{i}, b_{j}\right]^{m_{i j}}
$$

Зафиксируем пару индексов $u, v$ таких, что $m_{u v} \not \equiv 0(\bmod p)$. Пусть $Z_{p} * Z_{p}=$ $\langle a, b\rangle$. По лемме 1 отображение

$$
\begin{aligned}
a_{u} & \rightarrow a, \quad b_{v} \rightarrow b, \\
a_{i} \rightarrow 1 \text { при } i \neq u ; & b_{j} \rightarrow 1 \text { при } j \neq v
\end{aligned}
$$

продолжается до гомоморфизма $\varphi: G \rightarrow Z_{p} * Z_{p} \in \mathscr{M}$, при этом $g^{\varphi}=[a, b]^{m_{u v}} \neq 1$. Лемма доказана.

Следствие 1. Если $\mathscr{M} \subseteq \mathscr{R}_{p^{k}}-$ квазимногообразие групп такое, что $Z_{p^{k}} \in$ $\mathscr{M}$ и $Z_{p} * Z_{p} \in \mathscr{M}$, то $Z_{p^{s}} * Z_{p^{t}} \in \mathscr{M}$ при $s, t \leq k$.

Пусть $A_{r m}(r, m \geq 1)$ - группа, заданная в $\mathscr{R}_{p^{k}}$ порождающими

$$
a_{j l}, b_{j t} \quad(j=1, \ldots, 2 r ; l=1, \ldots, 2 m, t=1, \ldots, 2 w)
$$

и определяющими соотношениями

$$
\begin{gathered}
\prod_{j=1}^{w}\left[b_{1 j}, b_{1, w+j}\right] \prod_{j=1}^{m}\left[a_{1 j}, a_{1, m+j}\right]=\cdots=\prod_{j=1}^{w}\left[b_{2 r, j}, b_{2 r, w+j}\right] \prod_{j=1}^{m}\left[a_{2 r, j}, a_{2 r, m+j}\right], \\
{\left[a_{l j}, a_{t i}\right]=1 \quad(\text { при всех } l \neq t) .} \\
\text { Пусть } c_{1}=\prod_{j=1}^{w}\left[b_{1 j}, b_{1, w+j}\right] \prod_{j=1}^{m}\left[a_{1 j}, a_{1, m+j}\right] .
\end{gathered}
$$

Группы с таким же представлением, но относительно других квазимногообразий рассматривались в $[7,15,19]$, при $k=1$ - в [14].

Лемма 5. Пусть $\mathscr{M} \subseteq \mathscr{R}_{p^{k}}-$ произвольное квазимногообразие групп такое, что $Z_{p^{k}}, Z_{p} * Z_{p} \in \mathscr{M}$. Тогда $A_{r m} /\left\langle c_{1}\right\rangle, A_{r m} \in \mathscr{M}$.

ДокАЗАТЕЛьство. Пусть группа $B_{i}$ имеет в $\mathscr{R}_{p^{k}}$ представление

$$
B_{i}=\left\langle\left\{a_{i l}, b_{i t} \mid l=1, \ldots, 2 m, t=1, \ldots, 2 w\right\} ; \prod_{j=1}^{w}\left[b_{i j}, b_{i, w+j}\right] \prod_{j=1}^{m}\left[a_{i j}, a_{i, m+j}\right]=1\right\rangle .
$$

Эти группы изучались в $[16$, теорема $4.2 .24 ; 19]$ в многообразии $\mathscr{R}_{p}$. Доказательство того, что $B_{i} \in \mathscr{M}$ (индукцией по $m+w$ с применением леммы 4 ), является почти дословным повторением доказательств аналогичных утверждений из [16, теорема $4.2 .24 ; 19]$, поэтому его опустим. Группу $A_{r m} /\left\langle c_{1}\right\rangle$ можно собрать из групп $B_{i}$ с помощью повторения несколько раз конструкции, рассмотренной в лемме 4. По лемме $4 A_{r m} /\left\langle c_{1}\right\rangle \in \mathscr{M}$.

Рассмотрим гомоморфизм $\varphi: A_{r m} \rightarrow F_{2}$, при котором $b_{j 1}^{\varphi}=a, b_{j, w+1}^{\varphi}=$ $b(j=1, \ldots, 2 r)$, образы остальных порождающих равны единице. Существование $\varphi$ следует из теоремы Дика (лемма 1 ). Видим, что $c_{1}^{\varphi}=[a, b] \neq 1$. Следовательно, $\left\langle c_{1}\right\rangle \cap \operatorname{ker} \varphi=1$. Отсюда группа $A_{r m}$ вложима в группу $A_{r m} /\left\langle c_{1}\right\rangle \times$ $A_{r m} / \operatorname{ker} \varphi$, значит, $A_{r m} \in \mathscr{M}$. Лемма доказана.

Обозначим через $C_{p}^{t}(w)(1 \leq t \leq k-1)$ группу, имеющую в $\mathscr{R}_{p^{k}}$ представление

$$
C_{p}^{t}(w)=\left\langle a_{0}, a_{1}, \ldots, a_{2 w} ; a_{0}^{p^{t}} \prod_{i=1}^{w}\left[a_{i}, a_{w+i}\right]=1\right\rangle
$$


Лемма 6. В группе $C_{p}^{t}(w)$ коммутирование на нецентральных элементах транзитивно.

ДокАЗАТЕЛЬСтво. Несложно увидеть, что коммутант группы $C_{p}^{t}=C_{p}^{t}(w)$ - это свободная абелева группа экспоненты $p$ со свободными порождающими $\left[a_{i}, a_{j}\right](0 \leq i<j \leq 2 w)$. Пусть $a, b, c \notin Z\left(C_{p}^{t}\right)$ и $[a, b]=1,[a, c]=1$. Эти элементы могут быть представлены так:

$$
a=a_{0}^{k_{0}} a_{1}^{k_{1}} \ldots a_{2 w}^{k_{2} w} c_{1}, \quad b=a_{0}^{l_{0}} a_{1}^{l_{1}} \ldots a_{2 w}^{l_{2 w}} c_{2}, \quad c=a_{0}^{m_{0}} a_{1}^{m_{1}} \ldots a_{2 w}^{m_{2 w}} c_{3},
$$

где $c_{1}, c_{2}, c_{3}$ из коммутанта свободной группы. Тогда

$$
[a, b]=\prod_{0 \leq i<j \leq 2 w}\left[a_{i}, a_{j}\right]^{k_{i} l_{j}-k_{j} l_{i}}=1, \quad[a, c]=\prod_{0 \leq i<j \leq 2 w}\left[a_{i}, a_{j}\right]^{k_{i} m_{j}-k_{j} m_{i}}=1,
$$

откуда $k_{i} l_{j}-k_{j} l_{i} \equiv 0(\bmod p), k_{i} m_{j}-k_{j} m_{i} \equiv 0(\bmod p)$ для всех $0 \leq i<j \leq 2 w$. Отсюда следует, что ранг матрицы

$$
\left(\begin{array}{ccccc}
k_{0} & k_{1} & k_{2} & \ldots & k_{2 w} \\
l_{0} & l_{1} & l_{2} & \ldots & l_{2 w} \\
m_{0} & m_{1} & m_{2} & \ldots & m_{2 w}
\end{array}\right)
$$

равен 1. Следовательно, $l_{i} m_{j}-l_{j} m_{i} \equiv 0(\bmod p)$ для всех $0 \leq i<j \leq 2 w$. Это означает, что $[b, c]=1$. Лемма доказана.

Лемма 7. Если $w_{1}<w_{2}$, то при любом гомоморфизме $\varphi: C_{p}^{t}\left(w_{1}\right) \rightarrow$ $C_{p}^{s}\left(w_{2}\right)$ имеем $\left(a_{0}^{p^{t}}\right)^{\varphi}=1$.

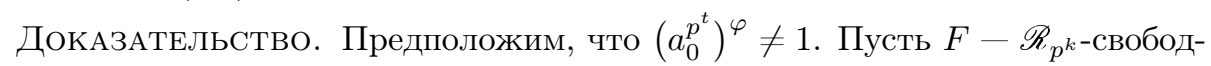
ная группа со свободными порождающими $a_{0}, a_{1}, \ldots, a_{2 w_{2}}, b_{i}$ - прообраз элемента $a_{i}^{\varphi}$ при естественном гомоморфизме группы $F$ на $C_{p}^{s}\left(w_{2}\right)$. Поскольку

$$
\left(a_{0}^{\varphi}\right)^{p^{t}} \prod_{i=1}^{w_{1}}\left[a_{i}^{\varphi}, a_{w_{1}+i}^{\varphi}\right]=1
$$

в $F$ справедливо равенство

$$
b_{0}^{p^{t}} \prod_{i=1}^{w_{1}}\left[b_{i}, b_{w_{1}+i}\right]=a_{0}^{m p^{s}} \prod_{i=1}^{w_{2}}\left[a_{i}, a_{w_{2}+i}\right]^{m}
$$

для некоторого $m(0 \leq m<p)$. Если $m=0$, то $b_{0}^{p^{t}} \in F^{\prime}$, откуда $b_{0}^{p^{t}}=1$. Значит, $\left(a_{0}^{p^{t}}\right)^{\varphi}=1$, что не так. Итак, предполагаем, что $m \neq 0$.

Рассмотрим группу $\bar{F}=F / F^{p^{2}}\left\langle a_{0}^{F}\right\rangle$, свободную в многообразии $\mathscr{R}_{p^{2}}$ со свободными порождающими $\bar{a}_{1}, \ldots, \bar{a}_{2 w_{2}}$. Пусть $\bar{b}_{i}-$ образ $b_{i}$ при естественном гомоморфизме $F$ на $\bar{F}$. Имеем

$$
\bar{b}_{i}^{p^{t}} \prod_{i=1}^{w_{1}}\left[\bar{b}_{i}, \bar{b}_{w_{1}+i}\right]=\prod_{i=1}^{w_{2}}\left[\bar{a}_{i}, \bar{a}_{w_{2}+i}\right]^{m} .
$$

Так как $\bar{b}_{i}^{p^{t}} \in \bar{F}^{\prime}(t \geq 1)$, то $\bar{b}_{i}^{p^{t}}=1$. Получили противоречие с тем, что, как доказано в $\left[16\right.$, теорема 4.2.3] (см. также $\left[6\right.$, лемма 3]), элемент $\prod_{i=1}^{w_{2}}\left[\bar{a}_{i}, \bar{a}_{w_{2}+i}\right]$ и, следовательно, любую его степень, отличную от единицы, нельзя записать в виде произведения меньше чем $w_{2}$ коммутаторов. Лемма доказана.

Следствие 2. Если $w_{1}<w_{2}$, то квазитождество

$$
\Phi_{t, w_{1}}=\left(\forall x_{0}\right) \ldots\left(\forall x_{2 w_{1}}\right)\left(x_{0}^{p^{t}} \prod\left[x_{i}, x_{w_{1}+i}\right]=1 \rightarrow x_{0}^{p^{t}}=1\right)
$$

истинно в группе $C_{p}^{s}\left(w_{2}\right)$. 


\section{§ 2. Группа $\boldsymbol{H}_{r m}$}

Зафиксируем квазимногообразия $\mathscr{M}$ и $\mathscr{N}, \mathscr{M} \varsubsetneqq \mathscr{N} \subseteq \mathscr{R}_{p^{k}}$. Предполагаем, что $\mathscr{M}$ порождается множеством конечных групп, в каждой из которых коммутирование на нецентральных элементах транзитивно. Кроме того, полагаем, что группы $Z_{p^{k}}, Z_{p} * Z_{p}$, принадлежат $\mathscr{M}$.

Если существует конечная группа из $\mathscr{N} \backslash \mathscr{M}$, заданная в $\mathscr{R}_{p^{k}}$ представлением, в котором все определяющие слова - элементы из коммутанта свободной группы (т. е. коммутаторные слова), то фиксируем группу $G \in \mathscr{N} \backslash \mathscr{M}$ с наименьшим числом таких соотношений. Если такой группы в $\mathscr{N} \backslash \mathscr{M}$ не существует, то в качестве фиксированной группы $G$ берем конечную группу из $\mathscr{N} \backslash \mathscr{M}$, в которой число соотношений в ее представлении (относительно $\mathscr{R}_{p^{k}}$ ) из формулировки леммы 3 наименьшее.

Итак, $G$ порождается элементами $x_{1}, \ldots, x_{n}$ и задается в $\mathscr{R}_{p^{k}}$ определяющими соотношениями $r_{1}=1, \ldots, r_{s}=1$, где

$$
\begin{gathered}
r_{1}=x_{1}^{p^{n_{1}}} w_{1}, \ldots, r_{d}=x_{d}^{p^{n_{d}}} w_{d}, \\
r_{d+1}=\left[x_{f(d+1)}, x_{g(d+1)}\right] w_{d+1}, \ldots, r_{s}=\left[x_{f(s)}, x_{g(s)}\right] w_{s},
\end{gathered}
$$

$n_{1}, \ldots, n_{d}$ - натуральные числа, отличные от нуля и меньшие $k, w_{1}, \ldots, w_{s}-$ элементы из коммутанта свободной группы.

Будем говорить, что группа $G$ обладает свойством $\left(P_{1}\right)$, если $r_{1}$ не принадлежит коммутанту свободной группы, и $G$ имеет свойство $\left(P_{2}\right)$, если все ее определяющие соотношения - элементы коммутанта свободной группы (т. е. коммутаторные слова). Отметим, что если $G$ обладает свойством $\left(P_{2}\right)$, то ее определяющие соотношения имеют вид

$$
\left[x_{f(1)}, x_{g(1)}\right] w_{1}=1,\left[x_{f(2)}, x_{g(2)}\right] w_{2}=1, \ldots,\left[x_{f(s)}, x_{g(s)}\right] w_{s}=1 .
$$

Можно и будем считать, что коммутатор $\left[x_{f(i)}, x_{g(i)}\right]$ входит в ненулевой степени только в слово $r_{i}$.

Возьмем произвольный неединичный элемент $v \in \mathscr{M}(G)$. Поскольку $G / G^{\prime}$ $\in \mathscr{M}$, то $\mathscr{M}(G) \subseteq G^{\prime}$. Если коммутатор $\left[x_{f(i)}, x_{g(i)}\right]$ входит в $v$ в ненулевой степени, то его можно исключить из записи элемента $v$ при помощи определяющего соотношения $r_{i}=1$. Таким образом, фиксируем элемент $v \in \mathscr{M}(G), v \neq$ $1, v=\prod_{i=1}^{q}\left[x_{h(i)}, x_{h(q+i)}\right]^{\gamma_{i}}, \gamma_{i} \neq \equiv 0(\bmod p)(i=1, \ldots, q)$, в запись которого входят коммутаторы $\left[x_{f(i)}, x_{g(i)}\right]$ в нулевой степени $(i=d+1, \ldots, s)$.

Пусть $H_{r m}(w)$ - группа (как правило, будем обозначать ее через $H_{r m}$ ), заданная в $\mathscr{R}_{p^{k}}$ порождающими $x_{1}, \ldots, x_{n}, a_{j l}, b_{j t}(j=1, \ldots, 2 r ; l=1, \ldots, 2 m$, $t=1, \ldots, 2 w)$ и следующими определяющими соотношениями:

(i) все соотношения группы $A_{r m}$;

(ii) $r_{1} c_{1}=1$;

(iii) если группа $G$ обладает свойством $\left(P_{1}\right)$, то в каждом коммутаторе $\left[x_{h(i)}, x_{h(q+i)}\right](i=1, \ldots q)$ фиксируем ровно один элемент (это $x_{h(i)}$ либо $\left.x_{h(q+i)}\right)$, отличный от $x_{1}$ (обозначим его через $\bar{x}_{i}$ ), и вводим соотношения: $\left[\bar{x}_{i}, b_{t j}\right]=1$, $\left[\bar{x}_{i}, b_{t, w+j}\right]=1$ для всех $j=1, \ldots, w$ и для всех $t$ таких, что $t \equiv i(\bmod q)$; если группа $G$ обладает свойством $\left(P_{2}\right)$, то вводим соотношения: $\left[x_{h(i)}, b_{t j}\right]=1$, $\left[x_{h(q+i)}, b_{t j}\right]=1$ для всех $j=1, \ldots, w$ и для всех $t$ таких, что $t \equiv i(\bmod q)$;

(iv) $r_{2}=1, \ldots, r_{s}=1$. 
Напомним, что $c_{1}=\prod_{j=1}^{w}\left[b_{1 j}, b_{1, w+j}\right] \prod_{j=1}^{m}\left[a_{1 j}, a_{1, m+j}\right]-$ слово из определения группы $A_{r m}$. Определение группы $H_{r m}$ зависит от того, каким из свойств $\left(P_{1}\right)$ либо $\left(P_{2}\right)$ обладает группа $G$.

Элемент $\prod_{i=1}^{q}\left[x_{h(i)}, x_{h(q+i)}\right]^{\gamma_{i}} \in H_{r m}$ снова будем обозначать через $v$.

Через $H$ всюду в работе будем обозначать группу, имеющую в $\mathscr{R}_{p^{k}}$ представление $H=\left\langle x_{1}, \ldots, x_{n} ; r_{2}=1, \ldots, r_{s}=1\right\rangle$.

Лемма 8. Справедливо следующее: $H \in \mathscr{M}$.

ДоказАтельство. Ясно, что $H /\left\langle r_{1}\right\rangle \cong G$, поэтому $H /\left\langle r_{1}\right\rangle \in \mathscr{N}$. Пусть $\langle a\rangle$ - циклическая группа порядка $p^{k}$. По лемме 1 отображение $x_{1} \rightarrow a, x_{i} \rightarrow 1$ $(i \geq 2)$ продолжаемо до гомоморфизма $\varphi: H \rightarrow\langle a\rangle \in \mathscr{N}$, при этом $r_{1}^{\varphi}=$ $a^{p^{n_{1}}}$. Группы $\left\langle r_{1}\right\rangle,\left\langle a^{p^{n_{1}}}\right\rangle$ имеют одинаковые порядки, равные $p^{k-n_{1}}$, поэтому $\varphi$ отображает $\left\langle r_{1}\right\rangle$ на $\left\langle a^{p^{n_{1}}}\right\rangle$ изоморфно. (Если $G$ обладает свойством $\left(P_{2}\right)$, то рассматриваем отображение $\varphi$, при котором $x_{f(1)} \rightarrow a \in F_{2}, x_{g(1)} \rightarrow b \in F_{2}, x_{i} \rightarrow$ 1 при $i \notin\left\{x_{f(1)}, x_{g(1)}\right\}$.) Видим, что $\left\langle r_{1}\right\rangle \cap \operatorname{ker} \varphi=1$. Значит, группа $H$ вложима в группу $H /\left\langle r_{1}\right\rangle \times H / \operatorname{ker} \varphi \in \mathscr{N}$. Поскольку определяющих соотношений у $H$ меньше, чем у $G$, то из выбора $G$ получаем, что $H \in \mathscr{M}$. Лемма доказана.

Лемма 9. Если для некоторого $w_{1}\left(1 \leq w_{1}<w\right)$ группы $C_{p}^{1}\left(w_{1}\right), C_{p}^{2}\left(w_{1}\right)$, $\ldots, C_{p}^{k-1}\left(w_{1}\right)$ принадлежат $\mathscr{M}$, то $H_{r m}(w) \in \mathscr{M} \vee q G$.

Доказательство. Ясно, что

$$
H_{r m} /\left\langle c_{1}\right\rangle \cong\left(G * A_{r m} /\left\langle c_{1}\right\rangle\right) / N
$$

где $N=\left\langle\left[\bar{x}_{i}, b_{t j}\right],\left[\bar{x}_{i}, b_{t, w+j}\right]\right| i=1, \ldots, q$, все $\left.t \equiv i(\bmod q), j=1, \ldots, w\right\rangle$ либо $N=\left\langle\left[x_{h(i)}, b_{t j}\right],\left[x_{h(q+i)}, b_{t j}\right]\right| i=1, \ldots, q, j=1, \ldots, w$, все $\left.t \equiv i(\bmod q)\right\rangle$, если $G$ обладает свойством $\left(P_{1}\right)$ либо $\left(P_{2}\right)$ соответственно.

По лемме 4 (примененной к квазимногообразию $\mathscr{M} \vee q G) H_{r m} /\left\langle c_{1}\right\rangle \in \mathscr{M} \vee q G$. Теперь достаточно построить гомоморфизм $\varphi: H_{r m} \rightarrow M$ в подходящую группу $M \in \mathscr{M}$ такой, что $c_{1}^{\varphi} \neq 1$.

Если $G$ обладает свойством $\left(P_{1}\right)$, то берем $M=C_{p}^{n_{1}}\left(w_{1}\right) \in \mathscr{M}$. Полагаем $x_{1}^{\varphi}=a_{0},\left(b_{t j}\right)^{\varphi}=a_{j},\left(b_{t, w+j}\right)^{\varphi}=a_{w_{1}+j}$ для $j=1, \ldots, w_{1}$ и для всех $t$. Образы остальных порождающих считаем равными единице. Поскольку $\bar{x}_{i}^{\varphi}=1$ при каждом $i$, видим, что применима лемма 1 , по которой отображение $\varphi$ продолжаемо до гомоморфизма (который снова обозначим буквой $\varphi$ ) группы $H_{r m}$ на группу $C_{p}^{n_{1}}\left(w_{1}\right)$, при этом $c_{1}^{\varphi}=\prod_{i=1}^{w_{1}}\left[a_{i}, a_{w_{1}+i}\right] \neq 1$.

Если $G$ обладает свойством $\left(P_{2}\right)$, то берем $M=F_{2}=\langle a, b\rangle \in \mathscr{M}$ и полагаем $x_{f(1)}^{\varphi}=a, x_{g(1)}^{\varphi}=b$. Если соотношение $\left[x_{f(1)}, b_{l 1}\right]=1$ или соотношение $\left[x_{g(1)}, b_{u 1}\right]=1$ присутствует среди определяющих соотношений группы $H_{r m}$, то полагаем $b_{l 1}^{\varphi}=a, b_{l, w+1}^{\varphi}=b^{-1}$ или $b_{u 1}^{\varphi}=b, b_{u, w+1}^{\varphi}=a$ соответственно. Если ни одно из соотношений $\left[x_{f(1)}, b_{t 1}\right]=1,\left[x_{g(1)}, b_{t 1}\right]=1$ не присутствует в списке (iii), то полагаем $b_{t 1}^{\varphi}=a, b_{t, w+1}^{\varphi}=b^{-1}$ для всех $t$. Образы остальных порождающих считаем равными единице. Поскольку коммутатор $\left[x_{f(1)}, x_{g(1)}\right]$ не входит в запись $v$, соотношения $\left[x_{f(1)}, b_{l 1}\right]=1,\left[x_{g(1)}, b_{l 1}\right]=1$ не могут одновременно присутствовать в списке определяющих соотношений группы $H_{r m}$. Это означает, что отображение $\varphi$ определено корректно. По лемме 1 это отображение продолжаемо до гомоморфизма (который снова обозначим буквой $\varphi$ ) на $F_{2}$, при этом $c_{1}^{\varphi}=[a, b]^{-1} \neq 1$. Лемма доказана. 
Из доказательства леммы 9 следует, что если группа $G$ задана только коммутаторными определяющими соотношениями, то предположение о наличии в $\mathscr{M}$ групп $C_{p}^{1}\left(w_{1}\right), C_{p}^{2}\left(w_{1}\right), \ldots, C_{p}^{k-1}\left(w_{1}\right)$ лишнее. В этом случае имеет место

Следствие 3. Если группа $G$ задана в $\mathscr{R}_{p^{k}}$ только коммутаторными определяющими соотношениями и $Z_{p^{k}}, Z_{p} * Z_{p} \in \mathscr{M}$, то $H_{r m} \in \mathscr{M} \vee q G$.

Лемма 10. Пусть $R-$ любая группа из $\mathscr{M}, \varphi: H_{r m} \rightarrow R-$ произвольный гомоморфизм и $r \geq q$. Тогда $v^{\varphi}=1$, где $v=\prod_{i=1}^{q}\left[x_{h(i)}, x_{h(q+i)}\right]^{\gamma_{i}} \in H_{r m}$. В частности, $H_{r m} \notin \mathscr{M}$.

ДокАЗАТЕЛЬСтво. Предположим, что $v^{\varphi} \neq 1$. Поскольку $\mathscr{M}$ порождается группами, в которых коммутирование на нецентральных элементах транзитивно, по признаку принадлежности (лемма 2 ) существует гомоморфизм $\psi$ группы $R$ в некоторую такую группу, при котором $v^{\varphi \psi} \neq 1$. Ввиду этого можно и будем считать, что коммутирование на нецентральных элементах группы $R$ транзитивно.

Так как $v^{\varphi} \neq 1$, найдется число $i(1 \leq i \leq q)$ такое, что $\left[x_{h(i)}, x_{h(q+i)}\right]^{\varphi} \neq 1$. $\mathrm{B}$ частности, $\bar{x}_{i}^{\varphi} \notin Z(R)$. Поскольку в группе $H_{r m}$ имеют место соотношения

$$
\left[\bar{x}_{i}, b_{t j}\right]=1, \quad\left[\bar{x}_{i}, b_{t, w+j}\right]=1 \quad(t \equiv i(\bmod q), j=1, \ldots, w)
$$

и коммутирование транзитивно на нецентральных элементах, получим

$$
\left[b_{t j}, b_{t, w+j}\right]^{\varphi}=1 \quad(t \equiv i(\bmod q), j=1, \ldots, w) .
$$

В случае, когда $G$ обладает свойством $\left(P_{2}\right)$, из соотношений

$$
\begin{gathered}
{\left[x_{h(i)}, x_{h(q+i)}\right]^{\varphi} \neq 1,\left[x_{h(i)}, b_{t j}\right]^{\varphi}=1,} \\
{\left[x_{h(q+i)}, b_{t j}\right]^{\varphi}=1 \quad(t \equiv i(\bmod q), j=1, \ldots, w)}
\end{gathered}
$$

и транзитивности коммутирования на нецентральных элементах следует, что $b_{t j}^{\varphi} \in Z(R)$, откуда также

$$
\left[b_{t j}, b_{t, w+j}\right]^{\varphi}=1 \quad(t \equiv i(\bmod q), j=1, \ldots, w) .
$$

Допустим, что существует $l$ такое, что $\left[a_{i l}, a_{i, m+l}\right]^{\varphi} \neq 1$. Возьмем любой коммутатор $\left[a_{t f}, a_{t, m+f}\right]^{\varphi}(t \neq i)$. Из определяющих соотношений группы $A_{r m}$ следует, что элементы, входящие в разные коммутаторы, перестановочные. Транзитивность коммутирования теперь влечет, что $\left[a_{t f}, a_{t, m+f}\right]^{\varphi}=1$. Вышеизложенное означает, что

$$
\prod_{j=1}^{m}\left[a_{t j}, a_{t, m+j}\right]^{\varphi}=1 \quad \text { при } t \equiv i(\bmod q) \quad(t \neq i),
$$

т. е. $c_{1}^{\varphi}=1$.

Если $\left[a_{i l}, a_{i, m+l}\right]^{\varphi}=1$ при любом $l$, то также получаем, что $c_{1}^{\varphi}=1$. Итак, доказано, что $c_{1}^{\varphi}=1$.

Пусть $\psi: H_{r m} \rightarrow H_{r m} /\left\langle c_{1}\right\rangle$ - естественный гомоморфизм. Поскольку $\left\langle c_{1}\right\rangle \subseteq \operatorname{ker} \varphi$, существует гомоморфизм $\xi: H_{r m} /\left\langle c_{1}\right\rangle \rightarrow R$ такой, что $\varphi=\psi \xi$, в частности, $\left(g\left\langle c_{1}\right\rangle\right)^{\xi}=g^{\varphi}$ для каждого элемента $g\left\langle c_{1}\right\rangle \in H_{r m} /\left\langle c_{1}\right\rangle$. При доказательстве леммы 9 заметили, что $H_{r m} /\left\langle c_{1}\right\rangle \cong\left(G * A_{r m} /\left\langle c_{1}\right\rangle\right) / N$. Это, в частности, означает, что $\left\langle x_{1}, \ldots, x_{n}\right\rangle^{\psi} \cong G$. Так как $v^{\psi} \in \mathscr{M}(G)$, заключаем, что $v^{\psi \xi}=1$, т. е. $v^{\varphi}=1$. Лемма доказана. 
Обозначим через $B_{r m}$ группу, заданную в $\mathscr{R}_{p^{k}}$ порождающими

$$
a_{j l}, b_{j t}(j=1, \ldots, 2 r ; l=1, \ldots, 2 m, t=1, \ldots, 2 w), \quad x_{1}, \ldots, x_{n}
$$

и определяющими соотношениями (это часть определяющих соотношений группы $\left.H_{r m}\right)$

$\left(i^{\prime}\right)\left[a_{l i}, a_{t j}\right]=1$ для всех $l, j, t, i$ таких, что $l \neq t$,

$\left(i i^{\prime}\right)\left[\bar{x}_{i}, b_{t j}\right]=1,\left[\bar{x}_{i}, b_{t, w+j}\right]=1$ для всех $t$ таких, что $t \equiv i(\bmod q)(j=$ $1, \ldots, w, i=1, \ldots, q)$, если группа $G$ обладает свойством $\left(P_{1}\right)$, и $\left[x_{h(i)}, b_{t j}\right]=1$, $\left[x_{h(q+i)}, b_{t j}\right]=1$ для всех $t$ таких, что $t \equiv i(\bmod q)(j=1, \ldots, w, i=1, \ldots, q)$, если группа $G$ обладает свойством $\left(P_{2}\right)$;

$\left(i i i^{\prime}\right) r_{2}=1, \ldots, r_{s}=1$.

Пусть $c_{t}=\prod_{j=1}^{w}\left[b_{t j}, b_{t, w+j}\right] \prod_{j=1}^{m}\left[a_{t j}, a_{t, m+j}\right] \in B_{r m}$ и

$$
N_{r m}=\left\langle r_{1} c_{1}, c_{1} c_{2}^{-1}, \ldots, c_{1} c_{2 r}^{-1}\right\rangle
$$

- подгруппа группы $B_{r m}$. Ясно, что $H_{r m} \cong B_{r m} / N_{r m}$.

Лемма 11. $B_{r m} \in \mathscr{M}$.

ДокАЗАТЕЛЬСтво. Пусть группа $B$ задана в $\mathscr{R}_{p^{k}}$ порождающими $a_{j l}, b_{j t}$ $(j=1, \ldots, 2 r ; l=1, \ldots, 2 m, t=1, \ldots, 2 w)$ и определяющими соотношениями $\left[a_{l j}, a_{t i}\right]=1$ для всех $l, j, t, i$ таких, что $l \neq t$ (это часть определяющих соотношений группы $\left.B_{r m}\right)$. По лемме $4 B \in \mathscr{M}$. Как легко заметить, $B_{r m}=(H * B) / N$, где

$$
N=\left\langle\left[\bar{x}_{i}, b_{t j}\right],\left[\bar{x}_{i}, b_{t, w+j}\right] \mid t \equiv i(\bmod q)(i=1, \ldots, q, j=1, \ldots, w)\right\rangle,
$$

если $G$ обладает свойством $\left(P_{1}\right)$, и

$$
N=\left\langle\left[x_{h(i)}, b_{t j}\right],\left[x_{h(q+i)}, b_{t j}\right] \mid t \equiv i(\bmod q)(i=1, \ldots, q, j=1, \ldots, w)\right\rangle,
$$

если $G$ обладает свойством $\left(P_{2}\right)$.

По лемме $8 H \in \mathscr{M}$, следовательно, по лемме $4 B_{r m} \in \mathscr{M}$. Лемма доказана.

Лемма 12. Пусть $B-l$-порожденная подгруппа группы $B_{r m}$. Если $m>$ $C_{l}^{2}=\frac{n(n-1)}{2}$, то $\Phi(B) \cap N_{r m}=1$, где $\Phi(B)$ - подгруппа Фраттини группы $B$.

ДоказАтельство. Пусть $g \in \Phi(B) \cap N_{r m}, g \neq 1$. Поскольку $\Phi(B)=B^{p} B^{\prime}$, элемент $g$ можно представить в виде $g=g_{1}^{p} c$ для некоторых элементов $g_{1} \in B$, $c \in B^{\prime}$. Так как $B-l$-порожденная группа, $B^{\prime}-C_{l}^{2}$-порожденная группа, следовательно, элемент $c$ можно записать в виде произведения $C_{l}^{2}$ коммутаторов. Таким образом, элемент $g$ имеет вид

$$
g=g_{1}^{p} \prod_{i=1}^{C_{l}^{2}}\left[f_{i}, f_{C_{l}^{2}+i}\right] .
$$

Так как $g \in N_{r m}$, то $g$ можно записать следующим образом:

$$
g=\left(r_{1} c_{1}\right)^{t_{1}} \prod_{i=2}^{2 r}\left(c_{1} c_{i}^{-1}\right)^{t_{i}} .
$$

Следовательно, $g$ можно представить так:

$$
g=r_{1}^{t_{1}} c_{1}^{t_{1}+t_{2}+\ldots} \prod_{i=2}^{2 r} c_{i}^{-t_{i}} .
$$


Поскольку $g \neq 1$, видим, что некоторый элемент $c_{i}$ (пусть для удобства это $\left.c_{1}\right)$ входит в $g$ в ненулевой степени. Пусть $F_{2 m}=\left\langle y_{1}, \ldots, y_{2 m}\right\rangle-$ свободная

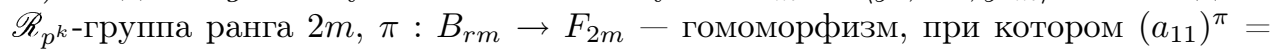
$y_{1}, \ldots,\left(a_{1,2 m}\right)^{\pi}=y_{2 m}$, образы остальных порождающих группы $B_{r m}$ равны 1 (существование такого гомоморфизма следует из леммы 1). Отсюда выводим, что

$$
\left(g_{1}^{\pi}\right)^{p} \prod_{i=1}^{C_{l}^{2}}\left[f_{i}^{\pi}, f_{C_{l}^{2}+i}^{\pi}\right]=\prod_{j=1}^{m}\left[\left(a_{1 j}\right)^{\pi},\left(a_{1, m+j}\right)^{\pi}\right]^{t_{1}+t_{2}+\ldots} .
$$

Так как в $F_{2 m}$ истинны квазитождества

$$
(\forall x)\left(\forall x_{1}\right) \ldots\left(\forall x_{2 n}\right) \quad\left(x^{p} \prod_{i=1}^{n}\left[x_{i}, x_{n+i}\right]=1 \rightarrow x^{p}=1\right), \quad n=1,2, \ldots,
$$

получаем в $F_{2 m}$ следующее равенство:

$$
\prod_{i=1}^{C_{l}^{2}}\left[f_{i}^{\pi}, f_{C_{l}^{2}+i}^{\pi}\right]=\prod_{j=1}^{m}\left[y_{j}, y_{m+j}\right]^{t_{1}+t_{2}+\ldots} .
$$

Это противоречит [16, теорема 4.2.3] (см. также [6, лемма 3]) тому, что элемент $\prod_{j=1}^{m}\left[y_{j}, y_{m+j}\right]$ и, следовательно, всякую его неединичную степень нельзя в $F_{2 m}$ записать в виде произведения меньшего чем $m$ числа коммутаторов. Лемма доказана.

Лемма 13. Пусть $A-l$-порожденная подгруппа группы $H_{r m}$. Если $m>$ $C_{l}^{2}$, то $A \in \mathscr{M}$.

ДокАЗАтЕЛЬство. Пусть $\varphi: B_{r m} \rightarrow H_{r m}$ - естественный гомоморфизм с ядром $N_{r m}, B$ - произвольный минимальный прообраз группы $A$ при гомоморфизме $\varphi$. (Аналогичный прием применялся в [20].) Покажем, что $B \cap N_{r m} \leq$ $\Phi(B)$.

Предположим, что это не так. Тогда $B \cap N_{r m} \nsubseteq M$ для некоторой максимальной подгруппы $M$ группы $B$. Отсюда $B=\left(B \cap N_{r m}\right) M$. Поскольку $\left(B \cap N_{r m}\right)^{\varphi}=1$, получаем, что $M^{\varphi}=A$. Это противоречит минимальности прообраза. Итак, $B \cap N_{r m} \leq \Phi(B)$.

По лемме $12 B \cap N_{r m}=1$, значит, $B \cong A$. Осталось воспользоваться леммой 11 , из которой следует, что $B \in \mathscr{M}$. Лемма доказана.

Смысл достаточно громоздких неравенств в лемме 14 следующий: числа, в ней встречающиеся «достаточно большие» или «одно намного больше другого» и $l=n+4 r m+4 r w$ - число порождающих группы $H_{r m}, n+4 w q+4 q-$ число порождающих группы $H_{q 1}$.

Лемма 14. Пусть $\varphi: H_{r m} \rightarrow H_{x y}$ - гомоморфизм, причем $y>C_{n+4 w q+4 q}^{2}$, $f=2^{\left|H_{x y}\right|}$. Положим $l=n+4 r m+4 r w$. Если $r>4 m q f$ либо $y>C_{l}^{2}$, то $v^{\varphi}=1$, где $v=\prod_{i=1}^{q}\left[x_{h(i)}, x_{h(q+i)}\right]^{\gamma_{i}}$ и $q$ - число коммутаторов в этом разложении.

ДокАЗАтЕЛьство. Пусть сначала $y>C_{l}^{2}$. Поскольку группа $H_{r m}$ является $l$-порожденной, $H_{r m}^{\varphi}$ также порождается $l$ элементами. Из леммы 13 следует, что $H_{r m}^{\varphi} \in \mathscr{M}$. В силу леммы $10 v^{\varphi}=1$. 
Предполагаем теперь, что $r>4 m q f$. Наша цель - построить гомоморфизм $\psi: H_{q 1} \rightarrow H_{x y}$ такой, что $v^{\psi}=v^{\varphi}$. Отметим, что $f$ больше числа подгрупп группы $H_{x y}$. Для каждого $i(1 \leq i \leq q)$ рассмотрим следующие подгруппы группы $H_{r m}$ :

$$
B_{i}=\left\langle a_{i, 1}, a_{i, 2}, \ldots, a_{i, 2 m}\right\rangle .
$$

Отметим, что подгруппы $B_{i}, B_{j}$ при $i \neq j$ перестановочны.

Пусть

$$
C_{t}=B_{t} B_{t+q}, B_{t+2 q} \ldots B_{t+(2 f-1) q} \quad(t=1,2, \ldots, q) .
$$

Существование всех этих групп $B_{i}$ и $C_{t}$ следует из неравенства $r>4 m q f$.

Исследуем $C_{t}^{\varphi}$. Поскольку число групп $B_{t}^{\varphi}, B_{t+q}^{\varphi}, B_{t+2 q}^{\varphi}, \ldots, B_{t+(f-1) q}^{\varphi}$ равно $f$, а число подгрупп группы $H_{x y}$ меньше $f$, то некоторые из этих подгрупп совпадают. Пусть $B_{t+q i}^{\varphi}=B_{t+q j}^{\varphi}(i<j)$. Из равенства $\left[B_{t+q i}, B_{t+q j}\right]=1$ следует

$$
1=\left[B_{t+q i}^{\varphi}, B_{t+q j}^{\varphi}\right]=\left[B_{t+q i}^{\varphi}, B_{t+q i}^{\varphi}\right],
$$

т. е. группа $B_{t+q i}^{\varphi}$ абелева. Поэтому

$$
c_{1}^{\varphi}=\prod_{u=1}^{w}\left[b_{t+q i, u}, b_{t+q i, w+u}\right]^{\varphi} \prod_{u=1}^{m}\left[a_{t+q i, u}, a_{t+q i, m+u}\right]^{\varphi}=\prod_{u=1}^{w}\left[b_{t+q i, u}, b_{t+q i, w+u}\right]^{\varphi} .
$$

Введем обозначения: $d_{t j}=b_{t+q i, j}^{\varphi}(j=1, \ldots, 2 w)$.

Сначала предположим, что $G$ обладает свойством $\left(P_{1}\right)$. Поскольку

$$
\left[\bar{x}_{t}, b_{t+q i, 1}\right]=1, \quad\left[\bar{x}_{t}, b_{t+q i, w+1}\right]=1,
$$

TO

$$
\left[\bar{x}_{t}^{\varphi}, d_{t j}\right]=1 \quad(j=1, \ldots, 2 w) .
$$

Аналогично, рассматривая подгруппы $B_{t+f q}^{\varphi}, B_{t+(f+1) q}^{\varphi}, B_{t+(f+2) q}^{\varphi}, \ldots, B_{t+(2 f-1) q}^{\varphi}$ группы $C_{t}^{\varphi}$, найдем элементы, обозначаемые через $d_{t+q, j}(j=1, \ldots, 2 w)$, из какой-нибудь из этих групп такие, что $\left[\bar{x}_{t}^{\varphi}, d_{t+q, j}\right]=1(j=1, \ldots, 2 w)$.

Пусть $G$ обладает свойством $\left(P_{2}\right)$. Так как

$$
\left[x_{h(i)}, b_{t+q i, 1}\right]=1, \quad\left[x_{h(q+i)}, b_{t+q i, 1}\right]=1,
$$

то

$$
\left[x_{h(i)}^{\varphi}, d_{t j}\right]=1, \quad\left[x_{h(q+i)}^{\varphi}, d_{t j}\right]=1 \quad(j=1, \ldots, 2 w) .
$$

Аналогично

$$
\left[x_{h(i)}^{\varphi}, d_{t+q, j}\right]=1, \quad\left[x_{h(q+i)}^{\varphi}, d_{t+q, j}\right]=1 \quad(j=1, \ldots, 2 w) .
$$

В обоих случаях

$$
c_{1}^{\varphi}=\prod_{u=1}^{w}\left[d_{t+q, u}, d_{t+q, w+u}\right] .
$$

Отсюда

$$
\prod_{u=1}^{w}\left[d_{t, u}, d_{t, w+u}\right]=\prod_{u=1}^{w}\left[d_{t+q, u}, d_{t+q, w+u}\right] .
$$

Воспользуемся леммой 1 , по которой отображение

$$
\begin{gathered}
b_{t j} \rightarrow d_{t j}, \quad b_{t+q, j} \rightarrow d_{t+q, j} \quad(t=1, \ldots, q, j=1, \ldots, 2 w), \\
x_{i} \rightarrow x_{i}^{\varphi}(i=1, \ldots, n), \quad a_{i j} \rightarrow 1
\end{gathered}
$$

продолжаемо до гомоморфизма $\psi: H_{q 1} \rightarrow H_{x y}$. Видим, что $v^{\psi}=v^{\varphi}$. Так как $H_{q 1}$ порождается $n+4 w q+4 q$ элементами и $y>C_{n+4 w q+4 q}^{2}$, по лемме 13 $H_{q 1}^{\psi} \in \mathscr{M}$. В силу леммы $10 v^{\psi}=1$, откуда $v^{\varphi}=1$. Лемма доказана. 
Теорема 1. Пусть $\mathscr{M}, \mathscr{N}$ - квазимногообразия групп, $\mathscr{M}$ порождается конечными группами, коммутирование на нецентральных элементах в которых транзитивно, $\mathscr{M} \varsubsetneqq \mathscr{N} \subseteq \mathscr{R}_{p^{k}}$. Предположим, что $Z_{p} *_{\mathscr{R}_{p}} Z_{p} \in \mathscr{M}$ и для некоторого $w_{1}$ группы $C_{p}^{1}\left(w_{1}\right), C_{p}^{2}\left(w_{1}\right), \ldots, C_{p}^{k-1}\left(w_{1}\right)$ принадлежат $\mathscr{M}$. Тогда промежуток $[\mathscr{M}, \mathscr{N}]$ в решетке квазимногообразий групп континуален.

ДоказАтельство. Возьмем конечную группу $G \notin \mathscr{M}, G \in \mathscr{N}$. Предполагаем, что среди групп из $\mathscr{N} \backslash \mathscr{M}$ группа $G$ задана наименьшим числом определяющих соотношений (относительно $\mathscr{R}_{p^{k}}$ ) таких, как в лемме 3.

Будем строить счетную последовательность групп $H_{1}, H_{2}, \ldots$, содержащихся в $\mathscr{N}$. Фиксируем любое число $w\left(w \geq w_{1}\right)$. Во всех в дальнейшем рассматриваемых группах $H_{r m}$ присутствует это $w$. В качестве $H_{1}$ берем произвольную группу $H_{r m}$ из $\mathscr{M} \vee q G$. Пусть группа $H_{i-1}$ уже построена. В качестве $H_{i}$ берем группу $H_{r_{i} m_{i}}$ такую, что

1) при любом гомоморфизме группы $H_{i}$ в группу $H_{j}(j<i)$ образ элемента $v$ равен единице;

$2)$ если $q_{i-1}$ - число порождающих группы $H_{i-1}$, то всякая $q_{i-1}$-порожденная подгруппа группы $H_{i}$ принадлежит квазимногообразию $\mathscr{M}$ (существование $H_{i}$ вытекает из лемм 13, 14).

Пусть $\mathbf{N}-$ множество всех натуральных чисел. Для подмножества $I \subseteq \mathbf{N}$ пусть $\mathscr{M}_{I}=q\left\{H_{i} \mid i \in I\right\}-$ квазимногообразие, порожденное всеми группами $H_{i}(i \in I)$. Если $H_{i} \in \mathscr{M}_{I}(i \notin I)$, то по признаку принадлежности (лемма 2) группа $H_{i}$ аппроксимируется группами из множества $\left\{H_{i} \mid i \in I\right\}$, что противоречит леммам 13,14 . Поэтому $H_{i} \notin \mathscr{M}_{I}$ при $i \notin I$, откуда получаем требуемое утверждение. Теорема доказана.

Присутствие групп $C_{p}^{1}\left(w_{1}\right), C_{p}^{2}\left(w_{1}\right), \ldots, C_{p}^{k-1}\left(w_{1}\right)$ в $\mathscr{M}$ нужно только в лемме 9. Если группа $G$ из доказательства теоремы 1 задана только коммутаторными определяющими соотношениями, то вместо леммы 9 можно воспользоваться следствием 3. Следствием доказательства теоремы 1 является

Теорема 2. Пусть квазимногообразие $\mathscr{M}$ порождается конечными группами, коммутирование на нецентральных элементах в которых транзитивно, $\mathscr{M} \subseteq \mathscr{R}_{p^{k}}$. Предположим, что $Z_{p^{k}}, Z_{p} * \mathscr{R}_{p^{k}} Z_{p} \in \mathscr{M}$. Если группу $G \notin \mathscr{M}$ можно задать в $\mathscr{R}_{p^{k}}$ только коммутаторными определяющими соотношениями, то промежуток $[\mathscr{M}, \mathscr{M} \vee q G]$ в решетке квазимногообразий групп континуален.

Теорема 3. Множество подквазимногообразий $\mathscr{R}_{p^{k}}(k \geq 2)$, порожденных конечной группой и не имеющих независимых базисов квазитождеств, бесконечно.

ДокАЗАТЕЛЬСтво. Пусть $\mathscr{M}_{w}$ - квазимногообразие, порожденное группами $C_{p}^{t}(w)(t=1, \ldots, k-1), Z_{p} * Z_{p}$, т. е. $\mathscr{M}_{w}=q\left(\left(\prod_{t=1}^{k-1} C_{p}^{t}(w)\right) \times\left(Z_{p} * Z_{p}\right)\right)$. При $w_{1}<w_{2}$ квазитождество $\Phi_{t, w_{1}}$ ложно в $C_{p}^{t}\left(w_{1}\right)$ и по следствию 2 истинно в каждой $C_{p}^{s}\left(w_{2}\right)$, значит, $\mathscr{M}_{w_{1}} \neq \mathscr{M}_{w_{2}}$ при $w_{1}<w_{2}$.

Если предположить, что $\mathscr{M}_{w}$ имеет независимый базис квазитождеств, то, как известно $[17$, предложение 6.3 .1$], \mathscr{M}_{w}$ имеет покрытие в решетке $L_{q}(\mathscr{K})$ квазимногообразий, содержащихся в $\mathscr{K}$, для любого конечно аксиоматизируемого квазимногообразия $\mathscr{K}$, содержащего $\mathscr{M}_{w}$. Но по теореме 1 любой промежуток $\left[\mathscr{M}_{w}, \mathscr{N}\right]$ континуален при $\mathscr{M}_{w} \varsubsetneqq \mathscr{N} \subseteq \mathscr{R}_{p^{k}}$, следовательно, $\mathscr{M}_{w}$ не имеет покрытий в $L_{q}\left(\mathscr{R}_{p^{k}}\right)$. Теорема доказана. 


\section{ЛИТЕРАТУРА}

1. Будкин А. И. Независимая аксиоматизируемость квазимногообразий групп // Мат. заметки. 1982. Т. 40, № 6. С. 817-826.

2. Будкин А. И. Независимая аксиоматизируемость квазимногообразий обобщенно разрешимых групп // Алгебра и логика. 1986. Т. 25, № 3. С. 249-266.

3. Медведев Н. Я. О квазимногообразиях $Z$-групп и групп // Сиб. мат. журн. 1985. Т. 26, № 5. С. 111-117.

4. Будкин А. И. О квазимногообразиях групп, не имеющих покрытий // Мат. заметки. 1985. Т. 37, № 5. С. 609-616.

5. Федоров А. Н. Квазитождества свободной 2-нильпотентной группы // Мат. заметки. 1986. Т. 40, № 5. C. 590-597.

6. Федоров А. Н. О подквазимногообразиях нильпотентных минимальных неабелевых многообразий групп // Сиб. мат. журн. 1980. Т. 21, № 6. С. 117-131.

7. Shakhova S. A. On the lattice of quasivarieties of nilpotent groups of class $2 / /$ Sib. Adv. Math. 1997. V. 7, N 3. P. 98-125.

8. Кравченко А. В., Нуракунов А. М., Швидефски М. В. О строении решеток квазимногообразий. І. Независимая аксиоматизируемость // Алгебра и логика. 2018. Т. 57, № 6. C. $684-710$.

9. Кравченко А. В., Нуракунов А. М., Швидефски М. В. О строении решеток квазимногообразий. II. Неразрешимые проблемы // Алгебра и логика. 2019. Т. 58, № 2. С. 179-199.

10. Adams M. E., Dziobiak W. Q-universal quasivarieties of algebras // Proc. Amer. Math. Soc. 1994. V. 120, N 4. P. 1053-1059.

11. Sapir M. V. The lattice of quasivarieties of semigroups // Algebra Univers. 1985. V. 21, N 2/3. P. $172-180$.

12. Шахова С. А. О квазимногообразии, порожденном конечной $p$-группой // Мат. заметки. 1993. T. 53, № 1. C. $144-148$.

13. Шахова С. А. К вопросу о мощности решетки квазимногообразий нильпотентных групп // Алгебра и логика. 1999. Т. 38, № 3. С. 368-375.

14. Будкин А. И. О решетке квазимногообразий нильпотентных групп // Алгебра и логика. 1994. Т. 33, № 1. С. 25-36.

15. Будкин А. И. О квазимногообразиях, содержащих нильпотентные группы без кручения // Алгебра и логика. 2001. Т. 40, № 6. С. 629-650.

16. Будкин А. И. Квазимногообразия групп. Барнаул: Изд-во Алт. ун-та, 2002.

17. Горбунов В. А. Алгебраическая теория квазимногообразий. Новосибирск: Науч. книга, 1999.

18. Мальцев А. И. Алгебраические системы. М.: Наука, 1970.

19. Федоров А. Н. Квазитождества конечных 2-нильпотентных групп. Деп. в ВИНИТИ. М., 1987. № 5489-В87.

20. Ольшанский А. Ю. Условные тождества в конечных группах // Сиб. мат. журн. 1974. T. 15 , № 6. C. 1409-1413.

Поступила в редакиию 24 января 2020 г. После доработки 5 мая 2020 г.

Принята к публикачии 17 июня 2020 г.

Будкин Александр Иванович

Алтайский государственный университет, ул. Ленина, 61, Барнаул 656064

budkin@math.asu.ru 